\title{
Chikungunya outbreaks from 2000 to 2015: a review
}

\begin{abstract}
Chikungunya is a mosquito-borne disease caused by an alphavirus from the Togaviridae family. The Chikungunya virus (CHIKV) is transmitted by the Aedesmosquitoes. The usual clinical signs of chikungunya are non-specific flu-like symptoms, a distinctive rash and severe joint pains. The disease shares some clinical signs with dengue and can be misdiagnosed in areas where dengue is endemic. There is neither vaccine nor a specific antiviral treatment for CHIKV. CHIKV was initially seen in the early 1950s at the boundary of Tanzania and Mozambique. For the past 5 decades, CHIKV was limited to sub-Saharan Africa in addition to Southeast Asia. The situation worsened when CHIKV re-emerged in Kenya in 2004 and reached several other countries in and around the Indian Ocean. The epidemic swiftly reached regions like India and Southeast Asia and transmission of CHIKV was reported for the first time in Europe in 2007 in Italy. The purpose of this review is to summarize CHIKV outbreaks that have been reported in the 15-year period from 2000 to 2015 to show that periodic outbreaks have occurred not only in Asia and Africa, but in Europe and the Americas. It is evident that CHIKV is not restricted to a single region but has become a global public health challenge. As many citizens from non-endemic countries visit areas endemic for CHIKV fever, medical professionals must learn to recognize such cases among travelers returning from such areas with non-specific symptoms such as fever, arthralgia and skin eruptions. In endemic areas for mosquito-borne diseases, clinicians must be educated about the recognition, diagnosis and timely reporting of chikungunya virus disease cases.
\end{abstract}

Keywords: chikungunya, outbreaks, Africa, Asia, Europe, America
Volume 2 Issue 6 - 2015

\author{
Doudou Diop,' Grigorij Meseznikov, ${ }^{2}$ Melvin \\ Sanicas $^{3}$ \\ 'EPLS Biomedical Research Center, Saint-Louis, Senegal \\ 2University Hospital Motol, Czech Republic \\ ${ }^{3}$ Bill and Melinda Gates Foundation, USA
} Correspondence: Doudou Diop, EPLS Biomedical Research
Center, Saint-Louis, Senegal,Tel +221775521929,

Email deuxdou@gmail.com

Received: September 25, 2015 | Published: November 25, 2015
Abbreviations: CHIKV, chikungunya virus; PAHO, pan American health organization; CDC, centers for disease control and prevention; WNF, west nile fever; MOHSA, ministry of health and social affairs; YF, yellow fever

\section{Introduction}

Chikungunya is a mosquito-borne disease caused by an alphavirus from the Togaviridae family. The Chikungunya virus (CHIKV) is transmitted by the Aedesmosquitoes, primarily Aedesaegyptiand Aedesalbopictus, the Asian 'Tiger' mosquito. Chikungunya virus breeds in the skin, moves to the liver, muscle, lymphoid tissue and brain, through the blood. The usual clinical signs of chikungunya are non-specific flu-like symptoms, a distinctive rash and severe joint pains, which may last for several weeks, months or even years after the acute phase of the infection. ${ }^{1}$ Complications of chikungunya include myocarditis, hepatitis, ocular and neurological disorders. ${ }^{2}$ Chikungunya disease was initially seen in 1952 in remote Africa at the boundary of Tanzania and Mozambique. Chikungunya illness is transferred by Aedes mosquitoes, it bear a resemblance to dengue and numerous other arboviral viruses, but is often related with arthralgia ${ }^{3}$ CHIKV causes significant morbidity and economic losses. ${ }^{4}$ For nearly 50 years, the disease was limited to sub-Saharan Africa in addition to Southeast Asia. The situation worsened when CHIKV re-emerged in Kenya in 2004and reached several other countries in and around the Indian Ocean. The epidemic swiftly reached regions like India and Southeast Asia and transmission of CHIKV was reported for the first time in Europe in 2007 in Emilia-Romagna in the north-east of Italy. Soon after, CHIKV infection autochthonous (indigenous) transmission was reported in south-eastern France, with 2 established cases in 2010 September. ${ }^{5}$ There is no vaccine or a specific antiviral treatment for CHIKV. The purpose of this review is to summarize CHIKV outbreaks that have been reported in the 15-year period from 2000 to 2015 to show that periodic outbreaks have occurred not only in Asia and Africa, but in Europe and the Americas. Although rates of chikungunya infection are variable globally, depending on outbreaks, continuous surveillance of the disease is crucial.

\section{Materials and methods}

The titles of journal articles in English and French published from 1 January 2000 through 30 April 2015, including the terms "chikungunya" in combination with "Europe" and "European", "Asia", "America", "Africa", "Australia" and the names of individual countries, "chikungunya" and "chikungunya outbreak" were searched. The search yielded $>1,000$ articles. These were screened to identify particular groups affected by chikungunya outbreaks and the public settings where chikungunya transmission occurred in the 15-year period from 2000 to 2015. In addition, the WHO, US CDC, PAHO, ProMED-mail websites were also reviewed for information and other literature (Figure 1).

\section{CHIKV in the Americas}

The first case of CHIKV infection was identified in 2010 in Rio de Janeiro, Brazil in an area high risk of dengue because of the presence of large numbers of A. aegypti mosquitoes, the same vector of CHIKV. ${ }^{6}$ The first autochthonous cases of CHIKV in the Western hemisphere was on the isle of Saint Martin in December 2013. Nearby documented infections have been established in 17 countries or districts in the Caribbean or South America (Guyana, , Anguilla, 
Antigua, French Guiana and Barbuda, Dominican Republic, British Virgin Islands, Dominica, Guadeloupe, Haiti, Martinique, Puerto Rico, Saint Barthelme, Saint Kitts and Nevis, Saint Lucia, Saint Martin, Saint Vincent and the Grenadines), where a great number of North American tourists regularly come on holidays; these tourists possibly can bring the risk of virus into the US. ${ }^{8}$ After 4months (around March 2014), around $>15000$ cases were recorded in 9 Caribbean islets in the French West Indies and the maximum recorded cases in South America occurred in French Guyana. There were cases in 15 atolls of the Caribbean at the end of April 2014 and the total number of cases reached 35,000 and 6 mortalities have been reported. ${ }^{8}$

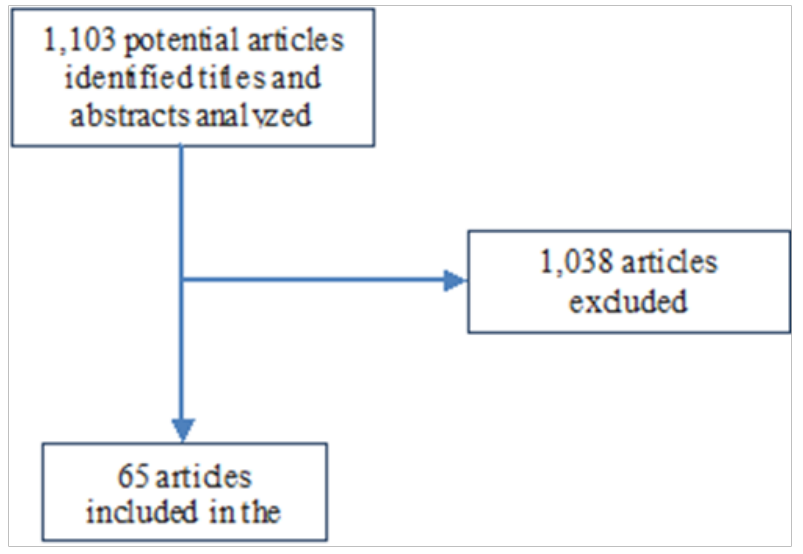

Figure I Flow chart for selection of studies.

In the United States, the epidemiology and cases of laboratoryconfirmed CHIKV infections were recorded from 2010 through 2013. Out of the 115 laboratory-confirmed CHIKV infections, 55 had known travel history, $53(96 \%)$ traveled to Asia while $2(4 \%)$ traveled to Africa. There were no locally-acquired infections. ${ }^{9}$ Two hundred thirty-two (232) imported cases of CHIKV infection were recorded in July2014 at the Nationwide Institute for Developing and Zoonotic Transmittable Diseases at the US Centers for Disease Control and Prevention (CDC). ${ }^{10}$ Two hundred seventy-two (272) imported cases and 11 locally acquired cases were identified in Florida ${ }^{8}$ including the first case of locally acquired Chikungunya. ${ }^{11}$ Several of these cases happened in the 14 or more regions that has Aedes a aegypti, as a local mosquito species. Furthermore, an obstinate vector mosquito, Aedesalbopictus, have been identified in 32 states over the past three decades. ${ }^{10}$ In March 2015, the US CDC reported that CHIKV infection had been identified in 44 countries or territories in the Caribbean, North, Central and South America and with more than 1.3million suspected cases and over 28,000 confirmed cases reported to the Pan American Health Organization (PAHO). ${ }^{12}$ The Isle of Saint Martin reported the highest incidence (115 cases per 1,000 population). Martinique (76 per 1,000), Saint Barthelemy $(74$ per 1,000$)$ and Guadeloupe (52 per 1,000) also reported high incidences of CHIKV infection. ${ }^{11,12}$

\section{CHIKV in Asia, Oceania and the pacific islands}

In Asia, CHIKV activity was first isolated in Bangkok, Thailand in 1958. Virus transmissions continued until 1964. The virus re-emerged in the mid-1970s and declined by $1976 .{ }^{13}$ India is known to be endemic to numerous infectious diseases. The major outbreak reported in India happened in Kolkata in 1963, which was followed by further outbreaks in Tamil Nadu Andhra Pradesh and Maharashtra in 1964 to 65 and in Barsi in 1973. CHIKV then appeared to have disappeared from India. The disease re-emerged in 2006 after a hiatus of 32 and triggered an outbreak that affected 13 provinces. The provinces that had an initial impact were Andhra Pradesh, Karnataka, Maharashtra, Madhya Pradesh, Tamil Nadu, Gujarat and Kerala. ${ }^{14}$ For the outbreak that started in the end of 2005, different sources have conflicting counts on the number of suspected cases ${ }^{15-17}$ ranging from hundreds of thousands up to a million. In the year 2008, almost 100,000 people in diverse communities of Kasargodu, Kerala were infected by CHIKV. An outbreak also occurred in Tirunelveli, Tamil Nadu in the year of 2009 to 2010. The CHIKV identified belongs to the Eastern Central Southern African genotype. ${ }^{18}$

Three cases of CHIKV infection were reported in Nepal in 2013. Physicians still do not consider CHIKV in the differential diagnosis; hence, CHIKV does not seem to be a public concern there as compared to neighbor India. ${ }^{19}$ Six cases of chikungunya were reported in native Bangladeshi women with ages ranging from 20 to 50 years. CHIKV is not that uncommon in Bangladesh. However, the presence of other arthropod-borne infections with the same courses of illness make medical doctors less likely to consider this infection..$^{20}$ After a 40 -year hiatus, CHIKV re-emerged in Sri Lanka in the latter part of 2006. A study of febrile patients in southern Sri Lanka reported that 3.5\% (28 out of 797) patients without acute dengue virus had chikungunya virus. Of these 18 out of 28 (64.3\%) were confirmed by viral isolation or real-time RT-PCR. ${ }^{21}$ CHIKV was reported for the first time in Bhutan in 2012. ${ }^{22}$

China's first documented outbreak of CHIK fever occurred in the Wanjiang District of Dongguan, Guangdong in September 2010. 253 cases were recorded, of which 129 were laboratory confirmed. The infection was imported. ${ }^{23}$ Taiwan reported 2 imported CHIKV cases identified by temperature screening at airports-1 infected with CHIKV East/Central/South African genotype returned from Singapore in 2006, the other infected with the Asian genotype returned from Indonesia in $2007 .{ }^{24}$ CHIKV has been a major problem in Southeast Asia with large numbers reported in Thailand, Malaysia and Singapore. ${ }^{25}$ In Singapore, where dengue has been endemic since the 1960s, the first CHIKV case was only reported in $2006^{26}$ while the first local outbreak occurred in January 2008. Since then, infection with the virus has been increasingly reported in the city-state. ${ }^{27}$ A study by the Ministry of Health and the National Environment Agency done within the period 2006 and 2009 showed that $812(75.7 \%)$ of the 1,072 cases recorded were indigenous. The main sources of importation were Malaysia and India with contract workers considered as source of the indigenous infections. ${ }^{28}$

Malaysia experienced its first CHIKV outbreak in Klang, Selangor in 1998 followed by an outbreak in the rural area of Perak in 2006 and in the urban areas of Johor in 2008. The first outbreak was due to an Asian genotype virus. ${ }^{29}$ The second outbreak was due to a Central/East African genotype virus. ${ }^{30}$ In 2009, CHIKV infection re-emerged in some states in Malaysia. A community-based case control study was carried out in the state of Kelantan. Among the 129 suspected cases, $54.4 \%$ were diagnosed to have CHIKV infection. ${ }^{31}$ A cross sectional study of CHIKV seroprevalence was carried out in 2009 in four states (Kuala Lumpur, Selangor, Pahang and Negeri Sembilan). From the 945 serum samples tested, 5.9\% was positive for CHIK IgG. ${ }^{32}$ A retrospective cross-sectional study done based on the database of clusters of patients with clinical diagnosis of chikungunya (CHIK) from January 2006 to December 2009 showed that of the 13,759 identified patients, $6,314(45.9 \%)$ were laboratory confirmed cases while 7,445 (54.1\%) were considered clinical cases by epidemiological link. ${ }^{33}$ 
In Thailand, there was a major outbreak of CHIKV fever between 2008 and 2009. This outbreak affected more than 46,000 individuals, the highest number of infections in any country in the region. ${ }^{34,35}$ In Indonesia, 1,901 febrile episodes were recorded between August 2000 to June 2004 and September 2006 to April 2008. Of these 135 (7.1\%) were CHIKV cases, providing an incidence rate of $10.1 / 1,000$ person years. CHIKV infections were identified all year round but showed increase in incidence during the rainy season (January to March). ${ }^{36}$ In Brunei Darussalam, the first cases of CHIKV were reported in 2011. The proactive surveillance system set up in January 2011 where all dengue-negative samples were tested for CHIKV, enabled identification of the first two detected cases and local transmission of CHIKV ${ }^{37}$ Brunei Darussalam is closer to Sabah but shares its borders with Sarawak, which experienced a large chikungunya outbreak in 2009 with 348 cases. Sabah reported no cases in 2009 but a surge in the number of cases was observed in the first part of $2010 .^{38}$

In Cambodia, the first case of CHIKV was detected in 1961. The virus again re-emerged in 2011 and a large outbreak occurred in the village of Trapeang Roka Kampong Speu Province in March 2012. ${ }^{39}$ A seroprevalence study of the village residents done in March 2012 reported that the outbreak affected families throughout the village and $44.7 \%$ of the population tested had evidence of CHIKV infection, which affected all age groups. ${ }^{40}$

In Japan, 14 patients at the Narita Airport Quarantine Station were laboratory-confirmed cases (using real-time RT-PCR) of imported CHIKV infection in 2013. ${ }^{41}$

In Yemen, an outbreak of dengue-like acute febrile illness was reported in Al Hodayda governorate in October 2010. By January 2011, a total of 1,542 cases had been recorded from 19 of the 26 districts in the governorate with 104 associated deaths. ${ }^{42}$ In Israel, a study including all serologically proven acute dengue and CHIKV infection cases recorded between 2008 and 2010 were analyzed-41 cases were diagnosed dengue while 15 cases were identified as CHIKV infection. Of the 15 CHIKV patients, $12(80 \%)$ lived in areas where A. albopictus mosquitoes were endemic. No autochthonous cases were reported. ${ }^{43}$

In Australia, 168 cases of CHIKV were imported between 2002 and 2012. Victoria and New South Wales had the largest number of reported cases. The main sources of the imported cases were Indonesia, India and Malaysia. There was a surge in the number of cases from 2008 to reach a peak in 2010. While Indonesia accounted for most of the CHIKV cases, travel from India had the highest CHIKV importation rate (number of imported cases per 100,000 travelers). ${ }^{44,45}$

\section{CHIKV in Europe}

CHIKV in India was the source of the outbreak in Italy. A local who travelled to India started the autochthonous transmission with native Aedesalbopictus mosquitoes. The epidemic curve of cases identified in Italy combined with detection of the virus in local Ae. albopictus mosquitoes showed that CHIKV infection was maintained by a mosquito-human-mosquito cycle. ${ }^{46}$ In 2006 , a surveillance system was established to be able to detect and manage dengue and CHIKV fever in areas with Aalbopictus to prevent secondary transmission. ${ }^{47}$ Between May and November 2007, 39 suspect cases were reported (25 imported and 14 autochthonous). Eight imported cases were confirmed, 1 CHIKV -the first documented autochthonous vectorborne transmission of CHIKV virus on the European continent. ${ }^{48}$

A large number of Chikungunya infection (231) cases were reported in Europe (Italy, France, Croatia and Madeira) throughout 2007 to 2012. CHIKV, endemic on the isle of Madeira, resulted into 122 hospitalizations with 1 death Emilia-Romagna, Italy. Through the Chikungunya outbreak in Italy and the dengue outbreak in Madeira, suitable actions for preventive measures and for the control of mosquitoes (Aedes aegypti and Aedesalbopictus) were efficiently applied. ${ }^{49}$ Although such an epidemic is new to Europe, it is probably caused by globalisation rather than climate change. Increased amounts of long distance tourism, travel and trade mean that organisms that live in and on people or goods have more opportunity to be transported across continents. ${ }^{50}$

In Veneto (northeastern part of Italy), a special integrated surveillance of summer fevers was established to promptly identify cases of West Nile Fever (WNF), dengue and CHIKV. Between 2010 and 2012, 234 patients with fever after travelling were identified, of which $27(11,5 \%)$ were found infected (24 with dengue and 3 with CHIKV).${ }^{51}$ Between January 2008 and October 2011, 130 cases of dengue / CHIKV were reported and $42.8 \%$ of CHIKV cases were imported from Indian Ocean Islands (Mauritius, Maldives, Bali and Sri Lanka). The estimated number of travelers exposed to CHIKV and dengue coming back to Italy was higher compared to notified cases, suggesting a potential underestimation of the real number of imported cases. $^{51,52}$

In October 2009, a French tourist returning from an island of Maldives was reported as the first case of CHIKV infection. ${ }^{53}$ Following the outbreak of autochthonous CHIKV fever in northern Italy, 2 independent cases of autochthonous transmission of chikungunya fever were identified in south-eastern France in September 2010. ${ }^{54}$ All the conditions for autochthonous transmission of CHIKV were present in southern Franc in the summer of 2014: a competent vector and a large number of travelers returning from the French Caribbean islands where an outbreak is occurring. From May to July 2014, there were 126 laboratory-confirmed imported chikungunya cases in mainland France. ${ }^{55}$ This particular species of mosquito has proliferated in Southern Europe and dispersion is likely to continue. ${ }^{56}$ In Spain, 10 cases of CHIKV infection were diagnosed in travelers returning from Haiti, the Dominican Republic or from both countries between the months April and June 2014. ${ }^{57}$ In Portugal, co-infection of dengue virus serotype 4 and CHIKV was reported in a woman in her early 50 s returning from Luanda, Angola, in January $2014 . .^{58}$

In Germany, where potential CHIKV vectors are not yet established, CHIKV infection is mandatorily notifiable. In September 2009, 2 cases of CHIKV infection were reported from German tourists who became ill after a trip to the Maldives. ${ }^{59}$ Cases from 2006 through 2009 were analyzed and 152 cases of symptomatic CHIKV infection were reported. Despite the fact that significant underdiagnoses is suspected, this analysis of CHIKV importation to Germany offers essential information about travel-associated CHIKV infection. The number of notified cases (17 to 53) per year means that CHIKV will be intermittently available for local transmission in as soon as a competent vector is established. ${ }^{60}$ In Belgium, 54 unpublished CHIKV cases have been reported from 2006 to 2008 (38 cases in 2006, 9 cases in 2007, 7 cases in 2008) primarily from tourists returning from countries with recent CHIKV epidemics such as Mauritius, Réunion Island, Sri Lanka, Madagascar and India. The first laboratory-confirmed, documented and published case of CHIKV was recorded in April 2009 in a Belgian tourist returning from Phuket, Thailand. ${ }^{61}$ 
Most Russian territory is in the Asian region but the case was recorded in the capital Russia so the investigators of the review included this in Europe. The case reported in September 2013 was that of an Indonesian visitor. The CHIKV strain CHIKVILEIVMoscow/1/2013 belonging to the Asian genotype was identified isolated and deposited into the Russian State Collection of viruses. ${ }^{62}$

\section{CHIKV in Africa}

CHIKV was initially identified and reported in Tanzania in 1952. A second outbreak occurred in South Africa in $1956 .{ }^{63}$ Thereafter, epidemics were recorded in Asia (Burma, Cambodia, India, Indonesia, Sri Lanka, Thailand, Timor, Vietnam, Philippines) and Africa (Angola, Burundi, Central African Republic, Democratic Republic of Congo, Guinea, Malawi, Nigeria, South Africa and Uganda) and through 1960's and 1990's. In June 2004, am epidemic started on Lamu Atoll, Kenya and spread to Comoros, La Reunion, Indian Ocean islands, resulting in nearly half a million reported cases. ${ }^{64}$ Between 2005 and 2007, all Southwestern Indian Ocean islands had on and off epidemics, except for Madagascar where persistent virus circulation was observed in an endemic-epidemic pattern. The virus was first detected in the spring of 2005 with only a few number of cases. By January 2006, there was a significant surge in the number of cases and at the peak of the epidemic; over 40,000 cases were reported per week. $.^{65} 266,000$ cases were estimated to have occurred during this outbreak and this was the first time that a number of neurological manifestations, fetal infections and mortality were reported to be associated with CHIKV. ${ }^{66-68}$ In 2009 and in 2010, 2 indigenous outbreaks were recorded in Reunion Island by the inter-epidemic surveillance system. ${ }^{68}$

In Kenya, 2 CHIKV outbreaks occurred beginning of 2004. The first outbreak in Lamu lead to an estimated 13,500 cases where greater than $70 \%$ of the population of the island were infected. ${ }^{69}$ The outbreak was initially thought to be caused by malaria but laboratory testing determined the cause to be CHIKV. Some months after the outbreak in Lamu, another outbreak happened in the city of Mombasa, the 2nd largest city in the country. Transmission of arboviral infection, including CHIKV, continues between known epidemics and remains unexceptional across Kenya. ${ }^{70}$

In Congo, a study conducted in 2011 analyzed 317 suspected cases of febrile illness characterized by severe joint pain. 37 (11.7\%) of the 317 cases were real-time RT-PCR-confirmed CHIKV patients. All tested negative for dengue. This was the first Chikungunya outbreak declared in the Republic of Congo. The seroprevalence study performed amongst blood donors before the outbreak provided evidence of previous CHIKV circulation. ${ }^{71}$ In June 2011, there were 7,014 cases in Brazzaville and 460 in Pool. By the end of June 2011 an IRIN representative reported that approximately 8,000 people were affected with CHIKV with no associated deaths. ${ }^{72}$

In a small study was conducted in Guinea in 2006 to identify arboviruses causing febrile diseases. ELISA and neutralization assays showed that $63 \%(30 / 47)$ of patients were infected with arboviruses, including 11 West Nile, 2 yellow fever, 1 dengue, 8 CHIKV and 5 Tahyna infections. Except for yellow fever, these are the first reported cases of human disease from these viruses in Guinea. ${ }^{73}$ A study in Northern Tanzania revealed that $7.9 \%$ of 870 febrile patients were PCR-confirmed acute CHIKV infections. ${ }^{74}$ Another study in the Mbeya Region was conducted to assess the epidemiological patterns of Alphavirusinfections. This region was not affected by the 2004
2007 outbreaks. However, investigators found that CHIKV or a closely related Alphavirus like o'nyong'nyong virus is circulating in the area. ${ }^{75}$

In Gabon, a simultaneous CHIKV and dengue outbreak was reported in two provinces (Ogooue Lolo and Haut Ogooue) from April to July 2010. A prospective study during the outbreak was done and 2,731 suspected cases were recorded in the two provinces and 1,208 cases $(44.2 \%)$ were laboratory confirmed. The study also revealed that the outbreak occurred during the rainy season and Aedesalbopictus was the main vector. ${ }^{76}$ In Cameroon, more than 400 cases of dengue-like fever were reported in Kumbo (northwest Cameroon) in 2006. A total of 105 sera were obtained from volunteers and there was serological evidence for recent CHIKV infection, as 54 subjects $(51.4 \%)$ had detectable IgM anti-CHIKV in their sera. No anti-ONNV and anti-DENV IgM antibodies were found amongst the sample tested. The findings suggest that the outbreak reported was due to $\mathrm{CHIKV} .{ }^{77} \mathrm{CHIKV}$ infection has been reported in West Africa since 1966, with the last outbreaks from Senegal in 1996 and 1997. ${ }^{78}$ In July 2012, researchers at the Mercy Hospital in Bo, Sierra Leone began a 1-year transmittable virus investigation to recognize which pathogens are instigating febrile sickness in the city. 400 (42.9\%) of 932 febrile patients were identified as positive for CHIKV by lateral flow immunoassay. ${ }^{79}$ Just recently, September 2015, the Ministry of Health and Social Affairs (MOHSA) of Senegal reported CHIKV circulation in the region of Kédougou. Samples were collected from 14 suspected cases for laboratory-confirmation at the Institute Pasteur of Dakar. Ten (10) of the 14 samples tested positive for CHIKV. ${ }^{80}$ In Sudan, an outbreak of hemorrhagic fever was recorded in South Kordofan from September to December 2005. Initial laboratory test results identified IgM antibodies against yellow fever (YF) virus and a yellow fever outbreak was declared. However, IgM antibodies to CHIKV were detected in five $(27 \%)$ cases. The results signify that both CHIKV and YF infections occurred during the outbreak.

\section{Discussion}

CHIKV was initially identified and reported in Tanzania in 1952. Epidemics were recorded in Africa and Asia through the years of 1960 's and 1990's. In Asia, CHIKV activity was first identified in Bangkok, Thailand in 1958. Imported cases among tourists coming from endemic areas have been identified in the Americas and several countries in Europe. The first transmission within continental Europe was reported from north-eastern Italy in August 2007. Thereafter, a large number of CHIKV infection cases were reported in Italy, France, Croatia and Madeira throughout 2007 to 2012. The first case of CHIKV infection in the Americas was identified in 2010 in Rio de Janeiro, Brazil while the first local transmission was identified in Caribbean countries and territories in 2013 (Figure 2).

It is evident that CHIKV is not restricted to a single region but has become a global public health challenge. Rapid diagnosis of CHIKV infections is of public health importance because the mosquito vectors are present in the various regions of the world. There is a real threat for the infection to spread in naïve population. The presence of a human population naïve to the chikungunya virus, competent vectors and the movement of people in and out endemic areas are factors that contribute to the extension of the circulation of the virus. Medical professionals should bear in mind that besides dengue, CHIKV should be considered as a differential diagnosis among travelers returning from Africa, Southeast Asia, the Caribbean region and the Indian 
subcontinent. The clinical picture of both dengue and CHIKV and even other viral infections can be similar and might be a challenge for medical professionals not familiar with these infections. Because of the risk of introduction into non-endemic countries, public health officials, medical professionals and the government need to be educated about the value of early recognition, prompt diagnosis and timely reporting of CHIKV cases.

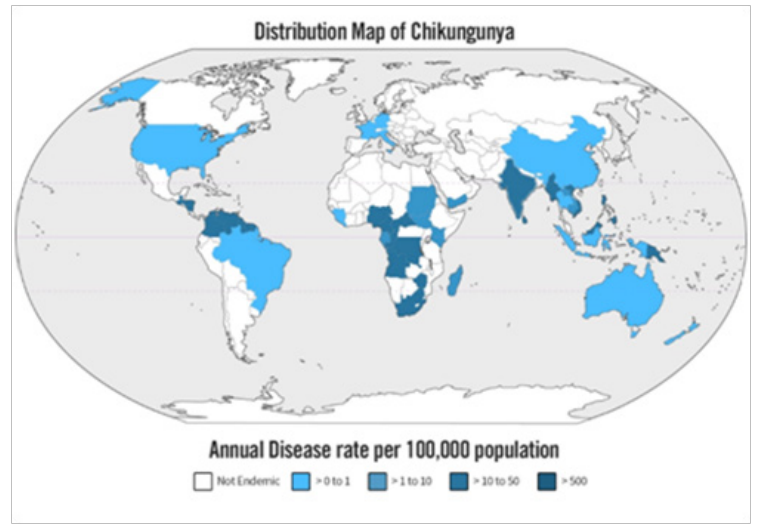

Figure 2 Global Distribution of Chikungunya

\section{Conclusion}

CHIKV outbreaks have been reported in Africa, Southeast Asia, the Caribbean region and the Indian subcontinent. In recent decades mosquito vectors have spread to Europe and the Americas. In 2007, transmission of diseases was reported for the first time in Europe. CHIKV infection has the possibility to spread into new areas in America and Europe where competent vectors are present. The huge majority of human inhabitants are immunologically naïve, which is a precondition for the circulation and spread of the virus. In the absence of an effective vaccine, rapid implementation of mosquito control measures and establishment of a system for continuous surveillance of the disease seems to be the only possible solution to prevent any such outbreak in the near future. Early identification of disease and control of vector will prevent the spread of disease. As many citizens from non-endemic countries visit areas endemic for CHIKV fever, medical professionals must learn to recognize such cases among travelers returning from such areas with fever, arthralgia and skin eruptions.

\section{Acknowledgements}

None.

\section{Conflict of interest}

The author declares no conflict of interest.

\section{References}

1. Pialoux G, Gaüzère BA, Jauréguiberry $\mathrm{S}$, et al. Chikungunya, an epidemic arbovirosis. Lancet Infect Dis. 2007;27(5):319-327.

2. Farnon EC, Sejvar JJ, Staples JE. Severe disease manifestations associated with acute chikungunya virus infection. Crit Care Med. 2008;36(9):2682-2683.

3. Thiberville SD, Moyen N, Dupuis Maguiraga L, et al. Chikungunya Fever Epidemiology, Clinical Syndrome, Pathogenesis and Therapy. Antiviral Res. 2013;99:345-370.
4. Pialoux G, Gauzere BA, Jaureguiberry S, et al. Chikungunya, an epidemic arbovirosis. Lancet Infect Dis. 2007;7:319-327.

5. Gould EA, GallianX P, de Lamballerie, et al. First cases of autochthonous dengue fever and Chikungunya fever in France: from bad dream to reality! Clin Microbiol Infect. 2010;16(12)1702-1704

6. Albuquerque IG, Marandino R, Mendonça AP, et al. Chikungunya virus infection: report of the first case diagnosed in Rio de Janeiro, Brazil. Rev Soc Bras Med Trop. 2012;45(1):128-129.

7. Khan K, Bogoch I, Brownstein JS, et al. Assessing The Origin of and Potential For International Spread of Chikungunya Virus From The Caribbean. PLos Curr. 2014:6.

8. Marc Fischer, Erin Staples. Arboviral Diseases Branch, National Center for Emerging and Zoonotic Infectious Diseases, CDC. Notes from the Field: Chikungunya Virus Spreads in the Americas-Caribbean and South America. Morbidity and Mortality Weekly Report. 2014;63(22):500501.

9. Lindsey NP, Prince HE, Kosoy O, et al. Chikungunya virus infections among travelers-United States, 2010-2013. Am J Trop Med Hyg. 2012;92(1):82-87.

10. PROMED-mail. Archive no. 20140519.2482881. Chikungunya (33): Caribbean.

11. M McCarthy. First case of locally acquired chikungunya is reported in US. BMJ. 2014;349:g4706.

12. PAHO. Chikungunya. 2014.

13. Fischer M, Staples JE. Notes from the field: chikungunya virus spreads in the Americas - Caribbean and South America, 2013-2014. MMWR Morb Mortal Wkly Rep. 2014;63(22):500-501.

14. Chhabra M, Mittal V, Bhattacharya D, et al. Chikungunya fever: a reemerging viral infection. Indian J Med Microbiol. 2008;26(1):5-12.

15. Disease outbreak news. Chikungunya and dengue in the southwest Indian Ocean, 17 March 2006. Geneva: WHO; 2006.

16. Kandath R. 1.5Lakh hit by Chikungunya. Deccan Herald.

17. Depoortere E, Coulombier D. Chikungunya risk assessment for Europe: recommendations for action. Euro surveill. 2006:11(19)

18. Cecilia D. Current status of dengue and chikungunya in India. WHO South-East Asia J Public Health. 2014;3(1):22-27.

19. Pun SB, Bastola A, Shah R. First report of Chikungunya virus infection in Nepal. J Infect Dev Ctries. 2014;8(6):790-792.

20. Hassan R, Rahman MM, Moniruzzaman M, Rahim A, Barua S, et al. Chikungunya-an emerging infection in Bangladesh: a case series. $J$ Med Case Rep. 2014;8:67.

21. Reller ME, Akoroda U, Nagahawatte A, et al. Chikungunya as a Cause of Acute Febrile Illness in Southern Sri Lanka. PLoS One. 2013;8(12):e82259.

22. Wangchuk S, Chinnawirotpisan P, Dorji T, et al. Chikungunya fever outbreak, Bhutan, 2012. Emerg Infect Dis. 2013;19(10):1681-16844.

23. Qiaoli Z, Jianfeng H, De W, et al. Maiden outbreak of chikungunya in Dongguan city, Guangdong province, China: epidemiological characteristics. PLoS One. 2012;7(8):e42830.

24. Shu PY, Yang CF, Su CL, et al. Two imported chikungunya cases, Taiwan. Emerg Infect Dis. 2008;14(8):1326-1327.

25. Pulmanausahakul R, Roytrakul S, Auewarakul P, et al. Chikungunya in Southeast Asia: understanding the emergence and finding solutions. Int $J$ Infect Dis. 2011;15(10):e671-e676. 
26. Leo YS, Chow AL, Tan LK, et al. Chikungunya outbreak, Singapore, 2008. Emerg Infect Dis. 2009;15(5):836-837.

27. Ng KW, Chow A, Win MK, et al. Clinical features and epidemiology of chikungunya infection in Singapore. Singapore Med J. 2009;50(8):785790 .

28. Ho K, Ang LW, Tan BH, et al. Epidemiology and control of chikungunya fever in Singapore. J Infect. 2011;62(4):263-270.

29. Kumarasamy V, Prathapa S, Zuridah H, et al. Re-Emergence of Chikungunya Virus in Malaysia. Med J Malaysia. 2006;61:221-225

30. Noridah O, Paranthaman V, Nayar SK, et al. Outbreak of Chikungunya Due to Virus of Central/East African Genotype in Malaysia. Med J Malaysia. 2007;62:323-328.

31. Yusoff AF, Mustafa AN, Husaain HM, et al. The assessment of risk factors for the Central/East African Genotype of chikungunya virus infections in the state of Kelantan:a case control study in Malaysia. BMC Infect Dis. 2013;13:211.

32. Azami NA, Salleh SA, Shah SA, et al. Emergence of chikungunya seropositivity in healthy Malaysian adults residing in outbreak-free locations:chikungunya seroprevalence results from the Malaysian Cohort. BMC Infect Dis. 2013;13:67.

33. Chua KB. Epidemiology of chikungunya in Malaysia: 2006-2009. Med J Malaysia. 2010;65(4):277-282.

34. Sasayama M, Benjathummarak S, Kawashita N, et al. Chikungunya virus was isolated in Thailand, 2010. Virus Genes. 2014;49(3):485-489.

35. Pulmanausahakul R, Roytrakul S, Auewarakul P, et al. Chikungunya in Southeast Asia: understanding the emergence and finding solutions. Int $J$ Infect Dis. 2011;15:e671.

36. Kosasih H, de Mast Q, Widjaja S, et al. Evidence for endemic chikungunya virus infections in Bandung, Indonesia. PLoS Negl Trop Dis. 2013;7(10):e2483.

37. Liew C, Yung CF. First detection of chikungunya infection and transmission in Brunei Darussalam. Singapore Med J. 2012;53(4):e66e68.

38. Chua KB. Epidemiology of Chikungunya in Malaysia: 2006-2009. Med J Malaysia. 2010;65:277-282.

39. Robinson M, Conan A, Duong V, et al. A model for a chikungunya outbreak in a rural Cambodian setting: implications for disease control in uninfected areas. PLoS Negl Trop Dis. 2014;8(9):e3120.

40. Furuichi M, Makie T, Honma Y, et al. Laboratory-confirmed dengue Fever and chikungunya Fever cases at the narita airport quarantine station in 2013. Jpn J Infect Dis. 2015;68(2):142-144.

41. Zayed A, Awash AA, Esmail MA, et al. Detection of Chikungunya virus in Aedesaegypti during 2011 outbreak in Al Hodayda, Yemen. Acta Trop. 2012;123(1):62-66.

42. Leshem E, Bin H, Shalom U, et al. Risk for emergence of dengue and chikungunya virus in Israel. Emerg Infect Dis. 2012;18(2):345-347.

43. Knope KE, Doggett SL, Kurucz N, et al. Arboviral diseases and malaria in Australia, 2011-12: annual report of the National Arbovirus and Malaria Advisory Committee. Commun Dis Intell Q Rep. 2014;38(2):E122E142.

44. Johnson DF, Druce JD, Chapman S, et al. Chikungunya virus infection in travellers to Australia. Med J Aust. 2008;188(1):41-43.

45. Staples J, Breiman R, Powers A. Chikungunya Fever: An Epidemiological Review of a Re-Emerging Infectious Disease. Clinical Infectious Diseases. 2009;49(6):942-948.

46. Gobbi F, Capelli G, Angheben A, et al. Human and entomological surveillance of West Nile fever, dengue and chikungunya in Veneto Region, Italy, 2010-2012. BMC Infect Dis. 2014;14:60.
47. Napoli C, Salcuni P, Pompa MG, et al. Estimated imported infections of Chikungunya and Dengue in Italy, 2008 to 2011. J Travel Med. 2012;19(5):294-297.

48. Tomasello D, Schlagenhauf P. Chikungunya and dengue autochthonous cases in Europe, 2007-2012. Travel Med Infect Dis. 2013;11(5):274-284.

49. Lines J. Chikungunya in Italy. BMJ. 2007;335(7620):576.

50. Rezza G, Nicoletti L, Angelini R, et al. Infection with Chikungunya virus in Italy: an outbreak in a temperate region. Lancet. 2007;370(9602):1840 1846.

51. Queyriaux B, Armengaud A, Jeannin C, et al. Chikungunya in Europe. Lancet. 2008;371(9614):723-724.

52. Receveur M, Ezzedine K, Pistone T, et al. Chikungunya infection in a French traveller returning from the Maldives, October, 2009. Euro Surveill. 2010;15(8):19494.

53. Gould EA, Gallian P, De Lamballerie X, et al. First cases of autochthonous dengue fever and chikungunya fever in France: from bad dream to reality! ClinMicrobiol Infect. 2010;16(12):1702-1704.

54. Paty MC, Six C, Charlet F, et al. Large number of imported chikungunya cases in mainland France, 2014:a challenge for surveillance and response. Euro Surveill. 2014;19(28):20856.

55. Sane J, Kurkela S, Vapalahti O. Chikungunya, a new global epidemic? Duodecim. 2011;127(5):457-463.

56. Requena-Méndez A, Garcia C, Aldasoro E, et al. Cases of chikungunya virus infection in travellers returning to Spain from Haiti or Dominican Republic, April-June 2014. Euro Surveill. 2014;19(28):20853.

57. Parreira R, Centeno-Lima S, Lopes A, et al. Dengue virus serotype 4 and chikungunya virus co-infection in a traveller returning from Luanda, Angola, January 2014. Euro Surveill. 2014:19(10).

58. Pfeffer M, Hanus I, Löscher T, et al. Chikungunya fever in two German tourists returning from the Maldives, September, 2009. Euro Surveill. 2010:15(13).

59. Frank C, Schöneberg I, Stark K. Trends in imported chikungunya virus infections in Germany, 2006-2009. Vector Borne Zoonotic Dis. 2011;11(6):631-636.

60. Bottieau E, Van Esbroeck M, Cnops L, et al. Chikungunya infection confirmed in a Belgian traveller returning from Phuket (Thailand). Euro Surveill. 2009:14(25).

61. Shchelkanov MIu, L'vov DK, Kolobukhina LV, et al. Isolation of the Chikungunya virus in Moscow from the Indonesian visitor (September, 2013). Vopr Virusol. 2014;59(3):28-34.

62. Jupp PG, Kemp A. What is the potential for future outbreaks of chikungunya, dengue and yellow fever in southern Africa? S Afr Med J. 1996;86(1):35-37.

63. Pialoux G, Gaüzère BA, Jauréguiberry S, et al. Chikungunya, an epidemic arbovirosis. Lancet Infect Dis. 2007;7(5):319-327.

64. Renault P, Balleydier E, D’Ortenzio E, et al. Epidemiology of Chikungunya infection on Reunion Island, Mayotte, and neighboring countries. Med Mal Infect. 2012;42(3):93-101.

65. Cire La Réunion-Mayotte. Epidemie de chikungunya a la Reunion: point au ler juin 2006 pour la semaine 21 allant du 22 au 28 mai 2006. 2006.

66. Borgherini G, Poubeau P, Staikowsky F, et al. Outbreak of chikungunya on Reunion Island: early clinical and laboratory features in 157 adult patients. Clin Infect Dis. 2007;44(11):1401-1407.

67. Ramful D, Carbonnier M, Pasquet M, et al. Mother-to-child transmission of chikungunya virus infection. Pediatr Infect Dis J. 2007;26(9):811815 . 
68. Sergon K, Njuguna C, Kalani R, et al. Seroprevalence of chikungunya virus (CHIKV) infection on Lamu Island, Kenya, October 2004. Am J Trop Med Hyg. 2008;78:333-337.

69. Sutherland LJ, Cash AA, Huang YJ, et al. Serologic evidence of arboviral infections among humans in Kenya. Am J Trop Med Hyg. 2011;85(1):158-161

70. Moyen N, Thiberville SD, Pastorino B, et al. First reported chikungunya fever outbreak in the republic of Congo, 2011. PLoS One. 2014;9(12):e115938.

71. Irin Chikv Update. CONGO: Chikungunya spreads to Pool region. IRIN 2011.

72. Jentes ES, Robinson J, Johnson BW, et al. Acutearboviral infections in Guinea, West Africa, 2006. Am J Trop Med Hyg. 2010;83(2):388-394.

73. Hertz JT, Munishi OM, Ooi EE, et al. Chikungunya and dengue fever among hospitalized febrile patients in northern Tanzania. Am J Trop Med Hyg. 2012;86(1):171-177.

74. Weller N, Clowes P, Dobler G, et al. Seroprevalence of alpha virus antibodies in a cross-sectional study in southwestern Tanzania suggests endemic circulation of chikungunya. PloS Negl Trop Dis 2014;8(7):e2979.
75. Nkoghe D, Kassa RF, Caron M, et al. Clinical forms of chikungunya in Gabon, 2010. PloS Negl Trop Dis. 2012;6(2):e1517.

76. Demanou M, Antonio-Nkondjio C, Ngapana E, et al. Chikungunya outbreak in a rural area of Western Cameroon in 2006:A retrospective serological and entomological survey. BMC Res Notes. 2010;3:128.

77. Pistone T, Ezzedine K, Boisvert M, et al. Cluster of chikungunya virus infection in travelers returning from Senegal, 2006. J Travel Med. 2009;16(4):286-288.

78. Ansumana R, Jacobsen KH, Leski TA, et al. Reemergence of Chikungunya Virus in Bo, Sierra Leone. Emerg Infect Dis. 2013;19(7):1108-1110.

79. Chikungunya-Senegal. WHO; 2015.

80. Gould LH, Osman MS, Farnon EC, et al. An outbreak of yellow fever with concurrent chikungunya virus transmission in South Kordofan, Sudan, 2005. Trans R Soc Trop Med Hyg. 2008;102(12):1247-1254. 